\title{
Design of Hybrid BCI using Multimodal EEG for Robotic Application
}

\author{
Pooja B. Kokare ${ }^{1}$, Prof. K. P. Wagh ${ }^{2}$, Prof. S.S. Savkare ${ }^{3}$ \\ Student, Department of Electronics and Telecommunication, JSPM Narhe Technical Campus, Pune, India ${ }^{1}$ \\ Professor, Department of Electronics and Telecommunication, JSPM Narhe Technical Campus, Pune, India ${ }^{2,3}$
}

\begin{abstract}
Over the past decade, many laboratories have begun to explore brain-computer interface (BCI) technology as a radically new communication option for those with neuromuscular impairments that prevent them from using conventional augmentative communication methods. BCl's provide these users with communication channels that do not depend on peripheral nerves and muscles. Moving something without touching. It has always something attractive for every person, speech recognition \& head movement is being the common methods. In recent technology consider imagination of people. The main thing has to control peripheral by brain activities. Electrical activity of brain is magic. A brain (BCI) is a system which allows direct translation of brain state into action. A BCI system works by extracting user brain signals, applying machine learning algorithm to classify the users brain state \&performing a computer controlled action.
\end{abstract}

Keywords: Brain computer interface (BCI), Mind machine interface (MMI), Electroencephalogram (EEG).

\section{INTRODUCTION}

The basic idea of $\mathrm{BCI}$ is to translate user produced patterns of brain activity into corresponding commands. A typical BCI is composed of signal acquisition and signal processing (including preprocessing, feature extraction and classification) [4]. Although some BCI systems do not include all components and others group two or three components into one Algorithm, most systems can be conceptually divided into signal acquisition, preprocessing, Feature extraction, and classification. Paralysis is usually caused by problems with the spinal cord that the brain uses to pass control messages to muscles.

Therefore, patients who are suffering from this severe problem need a great deal of support to enhance their ability to carry out daily activities. As a result, this problem has an impact on a person's quality of life and adds a high cost for the residential care packages since another person is needed to serve patient and satisfy his needs. In reviewing the literature, researchers are focusing on finding a technology that could be used by individuals who are affected by this problem. The goal was to develop devices that could be steered by the electrical activity of the brain using external electrodes attached to the user's scalp. BCI technology was envisioned as a promising and useful strategy that could give patients who are severely physically disabled new abilities to interact with the world around them through their mental activity. BCI is an emerging system that recognizes user brainwaves and reacts according to them. The system measures and analyses brain signals and then translate them into commands to control external devices such as wheelchair, $\mathrm{TV}$, and light system. Brainwaves are acquired by electroencephalography (EEG) sensors.
An electroencephalogram is a test to measure the electrical activity of the brain. This test is used as a non-invasive technology in which electrodes are implanted directly on the scalp.

\section{LITERATURE SURVEY}

The history of brain-computer interfaces (BCIs) starts with Hans Berger's discovery of the electrical activity of the human brain and the development of electroencephalography (EEG). In 1924 Berger was the first to record human brain activity by means of EEG. Berger was able to identify oscillatory activity, such as Berger's wave or the alpha wave $(8-13 \mathrm{~Hz})$, by analyzing EEG traces. Berger's first recording device was very rudimentary. He inserted silver wires under the scalps of his patients. These were later replaced by silver foils attached to the patients' head by rubber bandages. Berger connected these sensors to a Lippmann capillary electrometer, with disappointing results.

However, more sophisticated measuring devices, such as the Siemens double-coil recording galvanometer, which displayed electric voltages as small as one ten thousandth of a volt, led to success. Berger analyzed the interrelation of alternations in his EEG wave diagrams with brain diseases. EEGs permitted completely new possibilities for the research of human brain activities. Professor Jacques Vidal coined the term "BCI" and produced the first peerreviewed publications on this topic. Vidal is widely recognized as the inventor of BCIs in the BCI community, as reflected in numerous peer-reviewed articles reviewing and discussing the field. Vidal's first BCI relied on visual evoked potentials to allow users to control cursor 
direction, and visual evoked potentials are still widely oscillations necessarily synchronize at some instant during used in BCIs .After his early contributions, Vidal was not a memory process. The gamma oscillations phase locking active in BCI research, nor BCI events such as will then be the feature under study.Feature extraction is conferences, for many years. In 2011, however, he gave a the series of steps involved in extracting the information lecture in Graz, Austria, supported by the Future BNCI related to this feature. If, as in our above example, the project, presenting the first $\mathrm{BCI}$, which earned a standing feature is phase locking of the gamma oscillations, then ovation. Vidal was joined by his wife, Laryce Vidal, who we need instantaneous phase information about the signals previously worked with him on his first BCI project. Prof. under study, in the frequency range corresponding to Vidal will also present a lecture on his early BCI work at gamma oscillations. Using Classification the data the Sixth Annual BCI Meeting, scheduled for May-June obtained in the feature extraction stage, we try to detect 2016 at Asilomar, California.

\section{METHODOLOGY} signatures of the desired feature. Usually statistical tests are performed to obtain significance levels of the result.

Device Control component of a $\mathrm{BCI}$ is where the decision is finally translated into an act. After obtaining the

This project model aims to design a BCI system to control a service robot. An EEG amplifier is used to detect the EEG signals. EEG signals are sent to the pattern recognition module, and alpha rhythms are used as a switch to change the control command from SSVEP to motor imagery. Output of brain sensor is in electrical signal. This o/p is applied to computer vie Bluetooth for processing on them. MATLAB is used for processing on raw EEG signal. After processing data is applied towards microcontroller. After overall processing, PIC microcontroller sends command for robot.
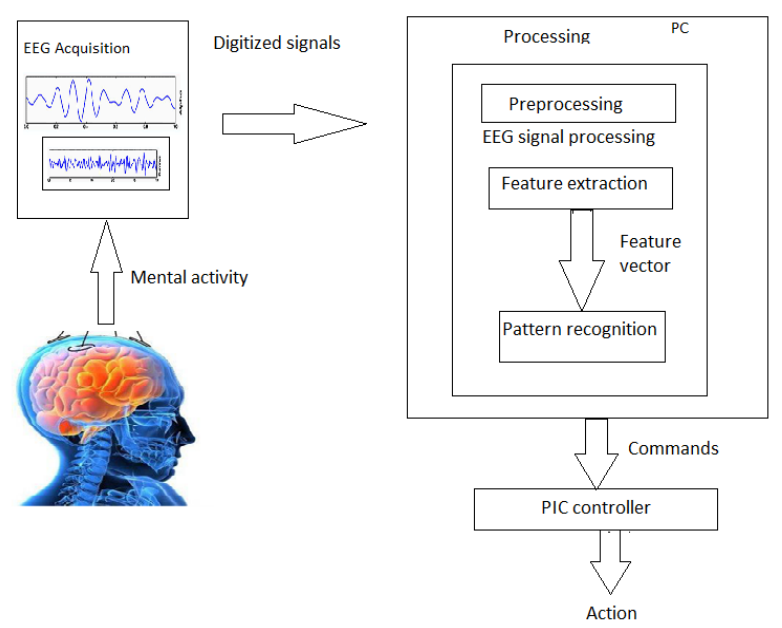

Fig. 1: Block diagram of the Hybrid BCI using multimodal EEG for Robotic application.

Signal acquisition and pre-processing is the basic step for any $\mathrm{BCI}$. It is the process of acquiring the brain signals in a form that we can read and analyze. First, an EEG, MEG, or any of those devices used to visualize the brain signals is involved. Then, the electronic signal is amplified and digitized (filtered and sampled). Feature extraction is most important in image reconization .Because the brain has more than millions of neural firings at every moment, researchers limit themselves to testing a certain aspect of the signals. This aspect under study is the "feature" considered as a basis for the BCI system. Every BCI is based on a certain hypothesis or assumption. For example a BCI that detects memory processes based on gamma oscillations phase locking, assumes that gamma decision from the classification stage, a control system implements the corresponding action. Output of brain sensor is in electrical signal. This $\mathrm{o} / \mathrm{p}$ is applied to computer vie Bluetooth for processing on them. MATLAB is used for processing on raw EEG signal. After proceeding data is applied towards microcontroller. After overall processing, PIC microcontroller send command for robot. The proposed system analyze the brain wave signals, and uses only single electrode headset based on EEG sensors which will monitor the EYE BLINKS, ATTENTION MODE, and MEDITATION MODE, and not going to monitor all the rays coming from the scalp, by analysing the frequencies ranges of the certain level, every human being will have different thoughts and emotions so it's enough monitor waves from forehead frontal point (FP1)alone, this mind wave headset sensor uses the Electromyography (EMG) Technique which will detect the muscle contractions that occurs while blinking the eye(i.e. rising the eyebrows or blinking)and this contraction will generate a unique electrical signal.[8] These electrical waves will be sensed by the brain wave sensor and it will convert the raw data into packets and transmit through wireless medium. Level analyzer unit (LAU) will receive the brain wave raw data and it will extract and process the signal using MATLAB platform. With this it's possible to control devices, according to the human thoughts both at attention and meditation modes, by only keeping single electrode on the forehead it is possible to do, since it's a portable headset device it can be easily operated by the elders.

\section{RESULT}

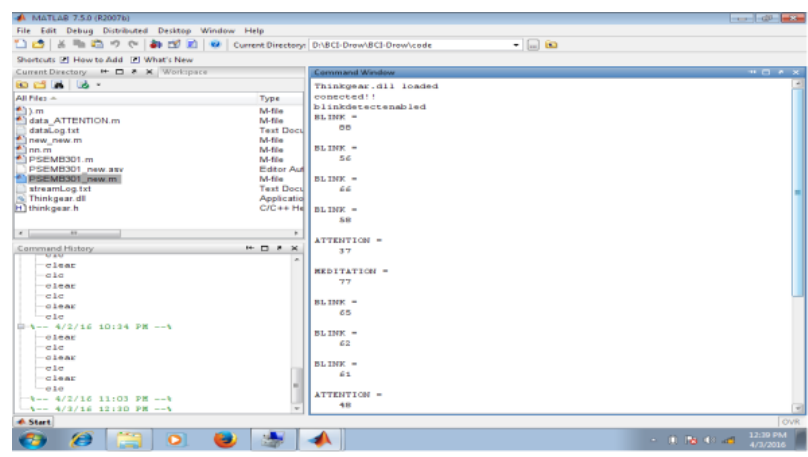

Fig no 2: Attention and Blinks values 
After getting these attention and blink values a graph will be generated and In the graph there will be two signals, the Black Signal is Blinking level and the red signal is Attention signal shown in figure 3 .

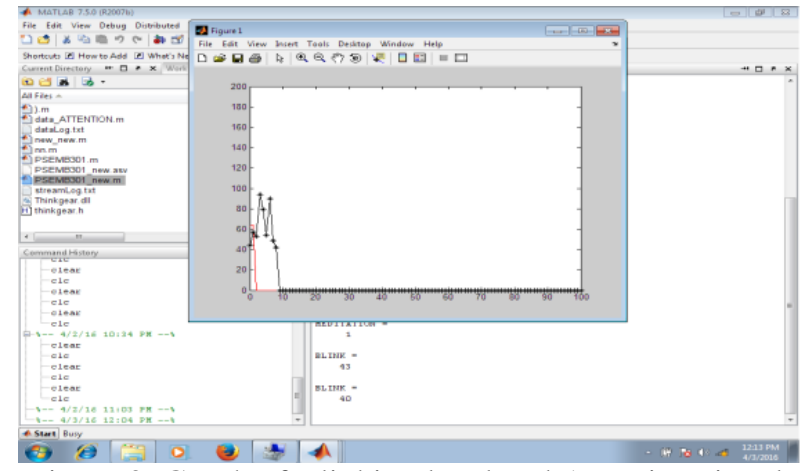

Fig no 3: Graph of Blinking level and Attention signal

If blinks are detected three times then robot moves towards right side. If blinks are more than three times then robot moves left side and for stop turn off the device.

\section{CONCLUSION}

In the recent daily life, not only disabled people but also elderly people need ane_ective BCI system to control a service robot to provide appropriate service for them. With advancing technological development, dierent BCI systems have been designed and used to control robot. Although current BCI systems can recognize and transfer the users intentions into control commands to move right or left . SSVEP or motor imagery is used to control a robot to move. Since the commands of these BCI systems are limited. Through incorporating the advantages of previous BCI systems, the bimodal EEG-based hybrid BCI system is proposed in this work. Considering the characteristics of evoked and the spontaneous EEG signals, this hybrid BCI system utilizes SSVEP signals,rhythm and feet motor imagery signals to generate mobility control commands.

\section{REFERENCES}

[1] Dario Assante and Claudio Fornaro "Involving graduating engineers in applying acommercial brain-computer interface to motorized wheelchair driving" 2015 IEEE Global Engineering Education Conference (EDUCON)

[2] Feng Duan, Dongxue Lin, Wenyu Li, and Zhao Zhang "Design of a MultimodalEEG-based Hybrid BCI System with Visual Servo Module" IEEE Transactions on Autonomous Mental Development,1943-0604 (c) 2015 IEEE.

[3] B.E. Swartz, E.S. Goldensohn, "Timeline of the history of EEG and associated fields," Electroencephalography and clinical Neurophysiology, vol. 106, No 2, pp. 173-176, 1998.

[4] L.F. Haas, H. Berger, "Richard Caton , "Journal of Neurology, Neurosurgery \& Psychiatry", Vol. 74, No 1, 2009.

[5] N. Birbaumer, "Just short of telepathy: can you interact with the outside world if you can't even blink an eye?", Psychology Today, May-June 2003.

[6] T.Karvinen, K.Karvinen, "Make a Mind-Controlled Arduino Robot", O'Reilly, 2011.

[7] M.Kurz, W.Almer, F.Landolt, "Brain Computer Interface", 2006
[8] K.Crowley, A.Sliney, I.Pitt, D.Murphy, "Evaluating a BrainComputer Interface to Categorise Human Emotional Response", IEEE 10th International Conference on Advanced Learning Technologies (ICALT), pp. 276-278, 5-7 July 2010.

[9] M.A.Garito, "Universities in Dialogue in a World without Distance", in Education Lanscapes in the $21^{\text {st }}$ Century: Crosscultural Challenges, 2010

[10] D Assante, R Sepe, "An international cooperation experience between the International Telematic University Uninettuno and the Helwan University: the double degree in ICT Engineeing", Global Engineering Education Conference (EDUCON), pp. 1011-1017, 2011.

[11] J. Naumann, "Search for Paradise: a Patient's Account of the Artificial Vision Experiment", Xlibris Corporation, 2012.

[12] K. Guðmundsdóttir, "Improving Players Control Over The Neuro Sky Brain Computer-Interface", Research report, School of Computer Science, 2011.

[13] J. Mostow, K. Chang, J. Nelso, Toward Exploiting EEG Input in a Reading Tutor, Arti_cial Intelligence in Education, 15th International Conference on Arti_cial Intelligence in Education, Auckland, July 2011.

[14] G. Rebolledo-Mendez, I. Dunwell, Assessing NeuroSky's Usability to Detect Attention Levels in an Assessment Exercise, International Conference on Human-Computer Interaction, pp 149-158, San Diego, 2009.

[15] http://www.google.com 